\title{
Spearman's hypothesis tested comparing Korean young adults with various other groups of young adults on the items of the Advanced Progressive Matrices - Erratum
}

Jan te Nijenhuis, Yu Yong Choi, Michael van den Hoek, Ekaterina Valueva and Kun Ho Lee

doi: 10.1017/S0021932019000026, Published by Cambridge University Press, April 2019.

The Acknowledgements section of this paper have been updated to more accurately reflect contributions to the article.

The Acknowledgements section of this paper should read as follows:

Acknowledgments. The authors would like to thank an anonymous reviewer and James Flynn for their constructive feedback which aided in improving the paper. 